University of Wollongong

Research Online

Faculty of Engineering and Information

Faculty of Engineering and Information

Sciences - Papers: Part A

Sciences

$1-1-2014$

\title{
Multi-polarization through-the-wall radar imaging using joint bayesian compressed sensing
}

Abdesselam Bouzerdoum

University of Wollongong, bouzer@uow.edu.au

Fok Hing Chi Tivive

University of Wollongong, tivive@uow.edu.au

Van Ha Tang

University of Wollongong, vht986@uowmail.edu.au

Follow this and additional works at: https://ro.uow.edu.au/eispapers

Part of the Engineering Commons, and the Science and Technology Studies Commons

Research Online is the open access institutional repository for the University of Wollongong. For further information contact the UOW Library: research-pubs@uow.edu.au 


\title{
Multi-polarization through-the-wall radar imaging using joint bayesian compressed sensing
}

\begin{abstract}
This paper presents a new image formation method for multi-polarization through-the-wall radar imaging. The proposed method combines wall clutter mitigation and scene reconstruction in a unified framework using multitask Bayesian compressed sensing. First, the radar signals are jointly recovered using Bayesian compressed sensing in the wavelet domain. Then, a subspace projection method is employed to mitigate the front wall reflections. This is followed by principal component analysis, which is used to compress the remaining wavelet coefficients and remove noise. A linear model is developed which relates the compressed wavelet coefficients directly to the image of the scene. For scene reconstruction, multitask Bayesian compressed sensing is further applied to simultaneously form the images associated with all polarimetric channels. Experimental results based on real radar data demonstrate that the proposed method improves image quality by enhancing target reflections and attenuating background clutter.
\end{abstract}

\section{Keywords}

radar, joint, bayesian, wall, compressed, polarization, multi, imaging, sensing

Disciplines

Engineering | Science and Technology Studies

\section{Publication Details}

A. Bouzerdoum, F. Tivive \& V. Tang, "Multi-polarization through-the-wall radar imaging using joint bayesian compressed sensing," in IEEE 19th International Conference on Digital Signal Processing (DSP), 2014, pp. 783-788. 


\title{
Multi-Polarization Through-the-Wall Radar Imaging using Joint Bayesian Compressed Sensing
}

\author{
Abdesselam Bouzerdoum, Fok Hing Chi Tivive, and Van Ha Tang \\ School of Electrical, Computer and Telecommunications Engineering \\ University of Wollongong, Wollongong, NSW 2522, Australia \\ Email: \{a.bouzerdoum, tivive, and vht986\}@uow.edu.au
}

\begin{abstract}
This paper presents a new image formation method for multi-polarization through-the-wall radar imaging. The proposed method combines wall clutter mitigation and scene reconstruction in a unified framework using multitask Bayesian compressed sensing. First, the radar signals are jointly recovered using Bayesian compressed sensing in the wavelet domain. Then, a subspace projection method is employed to mitigate the front wall reflections. This is followed by principal component analysis, which is used to compress the remaining wavelet coefficients and remove noise. A linear model is developed which relates the compressed wavelet coefficients directly to the image of the scene. For scene reconstruction, multitask Bayesian compressed sensing is further applied to simultaneously form the images associated with all polarimetric channels. Experimental results based on real radar data demonstrate that the proposed method improves image quality by enhancing target reflections and attenuating background clutter.
\end{abstract}

\section{INTRODUCTION}

Through-the-wall radar imaging (TWRI) is emerging as a viable sensing technology supporting a range of civilian and military applications, such as search-and-rescue, law enforcement, and urban surveillance and reconnaissance. It can be used for various purposes, including determining the building layout, and locating and identifying stationary objects behind walls. In the past years, numerous studies have been conducted in modeling and imaging stationary and moving targets behind walls and inside enclosed building structures [1]-[4]. However, there is still a need for producing high quality images that can effectively discriminate the targets of interest from clutter without increasing the data acquisition time.

Several TWRI studies have focused on the use of polarization of the electromagnetic (EM) waves for detecting targets or enhancing the discrimination of targets [5]-[11]. In [5], a method for through-the-wall detection of certain types of weapons was developed by analyzing the target signature in different polarizations. In [6], a polarimetric beamforming algorithm based on Green's function was proposed for TWRI applications. The formed images show the targets exhibit different characteristics with respect to the polarizations, and the wall clutter generated by the homogeneous wall does not appear in the cross-polarization images. In [7], an adaptive target detection technique using multi-polarization images was developed. The technique exploits the polarization diversity to improve detection. In [8], target segmentation and classification was achieved using features extracted from multipolarization images. In [9], a fully polarimetric scattering model of the human body was proposed for detecting a person behind walls. In [10] and [11], fuzzy logic based fusion techniques were proposed to combine multi-polarization images. In [12], a multi-polarization image formation method was developed for TWRI.

Most of multi-polarization TWRI studies assume that the wall reflections are removed, or at least significantly suppressed, prior to image formation. The problem of imaging with multiple polarizations is that the target may exhibit different responses when interrogated by different polarized signals. Furthermore, in practical applications, not all radar signals from the antenna array aperture can be acquired successfully due to occlusions by metallic obstacles or EM interferences. Therefore, the main concern of a practical fullpolarization TWRI system is to effectively reconstruct radar images with low background clutter and high target reflections from reduced polarimetric measurements.

Several techniques based on compressed sensing (CS) have been developed for TWRI [13]-[17]. The scene reconstruction is posed as an inverse problem where a linear model is employed which relates the compressive measurements from a single polarimetric channel directly to the imaged scene, which is assumed to be sparse. In [18], a method which combines wall clutter mitigation with CS image reconstruction was proposed. Wall clutter mitigation is performed on the space-frequency measurements using spatial filtering [19] or a subspace projection method [20]. The radar signals are recovered as range profiles from the reduced measurements. Then, following wall clutter mitigation, the same set of frequencies used to collect the initial measurements is employed for image formation. In [21], a method which performs simultaneous image formation and fusion of multiple polarization images was developed based on the multiple measurements vector (MMV) CS model. This CS-based method assumes that the same set of frequencies are collected across the antenna array aperture and polarizations.

In this paper, we propose an image formation method for multi-polarization TWRI. The proposed method combines wall clutter mitigation and image formation in a unified framework using multitask Bayesian compressed sensing. The rationale for using Bayesian compressed sensing is to exploit the statistical relationships among the collected measurements and the inter-channel correlations to reconstruct the images of all polarimetric channels. Furthermore, the proposed image 
formation method relaxes the constraint of having the same set of frequencies across the antenna array aperture. Another key property of the proposed image formation method is the use of a dimensionality reduction technique to compact the image formation model for fast and more accurate $\ell_{1}$-norm based scene reconstruction, without compromising the target information and image quality.

The remainder of the paper is organized as follows. Section II presents the TWRI signal model. Section III describes the proposed multi-polarization image formation method based on multitask Bayesian compressed sensing. Section IV presents the experimental results demonstrating the effectiveness of the proposed image formation method. Section $\mathrm{V}$ gives the concluding remarks.

\section{Multipolarization TWRI Signal Model}

This section describes the signal model of a through-thewall monostatic stepped-frequency radar with $L$ polarimetric channels. Consider an $N$-element linear array placed parallel to a wall at a certain standoff distance. The radar interrogates the scene behind the wall with a stepped-frequency signal of $M$ frequencies, equispaced over the desired bandwidth $\omega_{M}-$ $\omega_{1}$,

$$
\omega_{m}=\omega_{1}+(m-1) \Delta \omega, \quad m=1, \ldots, M,
$$

where $\omega_{1}$ is the lowest frequency in the frequency band and $\Delta \omega$ is the frequency step size. Assuming that the scene contains $P$ point-like targets, the radar signal of the $l$-th polarimetric channel received by the $n$-th antenna at the $m$-th frequency can be expressed as

$$
y_{\mathrm{t}, l}(m, n)=\sum_{p=1}^{P} \sigma_{p} \exp \left(-j \omega_{m} \tau_{n, p}\right),
$$

where $\sigma_{p}$ is the complex reflectivity of the $p$-th target and $\tau_{n, p}$ is the two-way propagation delay between the $n$-th antenna and the $p$-th target. Similarly, the signal reflected by a wall that behaves as a specular reflector can be expressed as [22]

$$
y_{\mathrm{w}, l}(m, n)=\sigma_{\mathrm{w}} \exp \left(-j \omega_{m} \tau_{\mathrm{w}}\right)
$$

where $\sigma_{\mathrm{w}}$ is the complex reflectivity of the wall and $\tau_{\mathrm{w}}$ is the two-way propagation delay between the wall and the $n$-th antenna. The parameter $\tau_{\mathrm{w}}$ is independent of the antenna position since the radar is placed parallel to the wall. Thus, the total baseband signal associated with the $l$-th polarization channel of the $m$-th frequency received by the $n$-th antenna is the superposition of the wall and target returns,

$$
y_{l}(m, n)=y_{\mathrm{w}, l}(m, n)+y_{\mathrm{t}, l}(m, n) .
$$

To describe the proposed CS-based method concisely, we rewrite the received signal given in Eq. (4) in matrix-vector form. Let $\boldsymbol{y}_{n, l}=\left[y_{l}(1, n), \ldots, y_{l}(M, n)\right]^{T}$ denote the vector containing the measurements received by the $n$-th antenna at the $l$-th polarization, where ${ }^{T}$ denotes the transpose operator. Assume that the imaged scene is divided into a rectangular grid consisting of $Q$ pixels. Let $s_{i, l}$ denote a weighted indicator function associated with the $l$-th polarization, defined as

$$
s_{i, l}= \begin{cases}\sigma_{p}, & \text { if the } p \text {-th target occupies the } i \text {-th pixel; } \\ \sigma_{\mathrm{w}}, & \text { if the wall occupies the } i \text {-th pixel; } \\ 0, & \text { otherwise. }\end{cases}
$$

Using the above weighted indicator function, we can form a column vector $s_{l}=\left[s_{1, l}, \ldots, s_{Q, l}\right]^{T}$ representing the imaged scene associated with the $l$-th polarization. Let $\tau_{n, q}$ denote the focusing delay between the $n$-th antenna and the $q$-th pixel, The radar signal of the $l$-th polarimetric channel received by the $n$-th antenna can be written in matrix-vector form as

$$
\boldsymbol{y}_{n, l}=\boldsymbol{\Psi}_{n} \boldsymbol{s}_{l}
$$

where $\boldsymbol{\Psi}_{n}=\left[\psi_{n}(m, q)\right]$ is an $M \times Q$ matrix with the $m q$ th element given by $\psi_{n}(m, q)=\exp \left(-j \omega_{m} \tau_{n, q}\right)$. Now, the relation between the measurements collected across the array aperture and the scene $s_{l}$ can be expressed as

$$
\widehat{\boldsymbol{y}}_{l}=\boldsymbol{\Psi} \boldsymbol{s}_{l},
$$

where $\widehat{\boldsymbol{y}}_{l}=\left[\boldsymbol{y}_{1, l}^{T}, \ldots, \boldsymbol{y}_{N, l}^{T}\right]^{T}$ and $\boldsymbol{\Psi}=\left[\boldsymbol{\Psi}_{1}^{T}, \ldots, \boldsymbol{\Psi}_{N}^{T}\right]^{T}$. The image associated with each polarization can be obtained by converting Eq. (7) into an L1-norm optimization problem and solving the problem using a sparse recovery method. However, this approach does not exploit the inter-channel correlations. In the next section, we describes the proposed CS-based image formation method for multi-polarization TWRI, which exploits the inter-channel correlations.

\section{Proposed Multi-Polarization Image Formation METHOD}

The proposed method consists of two stages: signal recovery followed by wall clutter mitigation, and scene reconstruction. The rationale for a two stage approach is to first recover the radar signal accurately in order to employ wall clutter mitigation before image formation in the second stage. In the first stage, the radar signal is modeled using a wavelet dictionary. Wall cutter mitigation is applied to the recovered wavelet coefficients in the first stage. The remaining coefficients are used for scene reconstruction in the second stage.

\section{A. Joint Radar Signal Recovery}

A set of frequencies are collected at each antenna location for signal recovery. The frequencies received by each antenna can be the same or different from one antenna to another. In [23], the radar signal is recovered as a range profile using Fourier dictionary. Here, we employ wavelet dictionary to accurately reconstruct the signal. Furthermore, we apply the multitask Bayesian compressed sensing (mt-BCS) technique [24] to exploit the signal correlations among the antennas.

Given a set of orthogonal wavelet basis functions $\mathbf{W} \in$ $\mathbb{R}^{M \times M}$, the signal $\boldsymbol{y}_{n, l}$ of Eq. (6) may be expressed as

$$
\boldsymbol{y}_{n, l}=\mathbf{W} \boldsymbol{x}_{n, l},
$$

where $\boldsymbol{x}_{n, l}$ is a vector of wavelet coefficients corresponding to the $n$-th antenna and the $l$-th polarization. To account for noise 
in the radar signal, the measurement vector $\boldsymbol{y}_{n, l}$ is modeled as

$$
\boldsymbol{y}_{n, l}=\mathbf{W} \boldsymbol{x}_{n, l}+\boldsymbol{e}_{n, l},
$$

where $\boldsymbol{e}_{n, l}$ is a zero-mean white Gaussian noise vector. According to CS theory, a subset of frequencies is sufficient to recover a sparse radar signal accurately. Thus, using the selection matrix $\phi_{n} \in \mathbb{R}^{J \times M}$ associated with the $n$-th antenna to choose $J$ frequencies, we can express the compressive measurement vector as

$$
\boldsymbol{z}_{n, l}=\boldsymbol{\phi}_{n} \boldsymbol{y}_{n, l}=\boldsymbol{\Phi}_{n} \boldsymbol{x}_{n, l}+\widetilde{\boldsymbol{e}}_{n, l}
$$

where $\boldsymbol{\Phi}_{n}=\phi_{n} \mathbf{W}$ and $\widetilde{\boldsymbol{e}}_{n, l}=\boldsymbol{\phi}_{n} \boldsymbol{e}_{n, l}$. For the problem at hand, the selection matrix $\phi_{n}$ has similar structure to an identity matrix, where each row contains only one nonzero element (equals 1) representing the selected frequency. We should note that since we are using real wavelet basis functions, the matrix $\boldsymbol{\Phi}_{n}$ is real. The complex vectors $\boldsymbol{z}_{n, l}, \boldsymbol{x}_{n, l}$ and $\widetilde{\boldsymbol{e}}_{n, l}$ are decomposed into real and imaginary parts to create the following composite vectors: $\widehat{\boldsymbol{z}}_{n, l}=$ $\left[\operatorname{Re}\left(\boldsymbol{z}_{n, l}\right)^{T}, \operatorname{Im}\left(\boldsymbol{z}_{n, l}\right)^{T}\right]^{T}, \widehat{\boldsymbol{x}}_{n, l}=\left[\operatorname{Re}\left(\boldsymbol{x}_{n, l}\right)^{T}, \operatorname{Im}\left(\boldsymbol{x}_{n, l}\right)^{T}\right]^{T}$ and $\widehat{\boldsymbol{e}}_{n, l}=\left[\operatorname{Re}\left(\widetilde{\boldsymbol{e}}_{n, l}\right)^{T}, \operatorname{Im}\left(\widetilde{\boldsymbol{e}}_{n, l}\right)^{T}\right]^{T}$, where Re and Im denote the real and imaginary operators, respectively. Accordingly, the measurement matrix $\widehat{\boldsymbol{\Phi}}_{n}$ can be represented as

$$
\widehat{\boldsymbol{\Phi}}_{n}=\left[\begin{array}{cc}
\boldsymbol{\Phi}_{n} & \mathbf{0} \\
\mathbf{0} & \boldsymbol{\Phi}_{n}
\end{array}\right] \text {. }
$$

The mt-BCS technique models the likelihood of the vector $\widehat{\boldsymbol{x}}_{n, l}$ as a multivariate Gaussian function,

$$
p\left(\widehat{\boldsymbol{z}}_{n, l} \mid \widehat{\boldsymbol{x}}_{n, l}, \boldsymbol{\alpha}_{\mathbf{0}}\right)=(2 \pi / \theta)^{-J / 2} \exp \left(-\frac{\theta}{2}\left\|\widehat{\boldsymbol{z}}_{n, l}-\widehat{\boldsymbol{\Phi}}_{n} \widehat{\boldsymbol{x}}_{n, l}\right\|^{2}\right),
$$

where $\boldsymbol{\alpha}_{\mathbf{0}}$ is the noise precision. Let $\widehat{x}_{n, l}(i)$ denote the $i$-th wavelet coefficient associated with the $n$-th antenna and $l$-th polarization. The wavelet coefficients $\widehat{x}_{n, l}(i), i=$ $1,2, \ldots, 2 R$, are assumed to be drawn from a product of zero mean Gaussian distributions that are shared by all $N$ antennas. Thus, we have

$$
p\left(\widehat{\boldsymbol{x}}_{n, l} \mid \boldsymbol{\alpha}, \boldsymbol{\alpha}_{\mathbf{0}}\right)=\prod_{i=1}^{2 R} \mathcal{N}\left(\widehat{x}_{n, l}(i) \mid 0, \alpha_{i}^{-1}\right),
$$

where $\mathcal{N}\left(\widehat{x}_{n, l}(i) \mid 0, \alpha_{i}^{-1}\right)$ is a zero-mean Gaussian function with precision $\alpha_{i}$. Note that the parameters $\left\{\alpha_{i}\right\}_{i=1,2, \ldots, 2 R}$ are shared among all antennas, which, in this sense, allows the recovery of the wavelet coefficients to be statistically related. In [25], a modified mt-BCS technique was proposed to solve complex problems, which will be used to recover the radar signals for all antennas and form the images for all polarimetric channels. However, before image formation we need to identify and remove the wavelet coefficients associated with the wall returns.

\section{B. Wall Clutter Mitigation}

The radar signal can be recovered from Eq. (8) using the estimated wavelet coefficients. Then, a wall clutter mitigation method, such as spatial filtering [19] or entropy-based method
[26] can be used to suppress the wall reflections. Here, we apply the subspace method described in [27] directly to the wavelet coefficients. Let $\mathbf{X}$ denote the matrix containing the wavelet coefficients recovered from all antennas and polarimetric channels, $\mathbf{X}=\left[\boldsymbol{x}_{1,1}, \boldsymbol{x}_{2,1}, \ldots, \boldsymbol{x}_{N, L}\right]$. Using SVD, the matrix $\mathbf{X}$ can be decomposed as

$$
\mathbf{X}=\mathbf{U} \boldsymbol{\Sigma} \mathbf{V}^{H}
$$

where ${ }^{H}$ denotes the Hermitian transpose, $\mathbf{U}=\left[\boldsymbol{u}_{1}, \ldots, \boldsymbol{u}_{L}\right]$ and $\mathbf{V}=\left[\boldsymbol{v}_{1}, \ldots, \boldsymbol{v}_{N}\right]$ are unitary matrices containing the left and right singular vectors, respectively, and $\Sigma$ is a rectangular matrix containing the singular values along the main diagonal arranged in descending order. Since the wall reflections are relatively stronger than the target reflections, they mostly reside in the first few singular vectors associated with the dominant singular values [20]. Therefore, we define the wall subspace as

$$
\mathbf{S}_{\mathrm{w}}=\sum_{i \in \mathcal{D}} \boldsymbol{u}_{i} \boldsymbol{v}_{i}^{H}
$$

where $\mathcal{D}$ denotes the index set of the singular vectors spanning the wall subspace. The singular vectors capturing the strong wall reflections are determined by applying a threshold technique to the singular value spectrum [27]. To remove the wavelet coefficients associated with the wall returns, the matrix $\mathbf{X}$ is projected onto a subspace orthogonal to the wall subspace

$$
\widetilde{\mathbf{X}}=\left(\mathbf{I}-\mathbf{S}_{\mathrm{w}} \mathbf{S}_{\mathrm{w}}^{H}\right) \mathbf{X},
$$

where $\mathbf{I}$ is the identity matrix. After the projection operation, the wavelet coefficients may still contain some wall residuals. Thus, we transform the wavelet coefficients into radar signals and compute their corresponding range profiles by first tapering the signal with a window function and then applying the inverse fast Fourier transform. We employ a segmentation technique described in [27] on the range profile to mitigate the wall residuals. Then, the range profiles are transformed back to the wavelet domain to obtain the wavelet coefficients carrying the target information.

\section{Simultaneous Multipolarization Image Formation}

The scene can be reconstructed by first recovering the radar signal using the inverse wavelet transform, see Eq. (8), and then applying a backprojection method, similar to the approach described in [23]. Here, we formulate a linear model that maps the wavelet coefficients directly to the imaged scene and solve jointly the multi-polarization image formation problem using the mt-BCS technique.

Let $\widetilde{\boldsymbol{x}}_{n, l}, n=1,2, \ldots, N, l=1,2, \ldots, L$ be the remaining wavelet coefficients after removing those associated with the wall returns. Using Eqs. (8) and (6), we can express these wavelet coefficients as

$$
\widetilde{\boldsymbol{x}}_{n, l}=\mathbf{W}^{T} \boldsymbol{\Psi}_{n} \widetilde{\boldsymbol{s}}_{l},
$$

where $\widetilde{\boldsymbol{s}}_{l}$ is the target scene associated with the $l$-th polarization. The new measurement vectors, $\widetilde{\boldsymbol{x}}_{n, l}$, obtained from all antenna locations for a single polarimetric channel, can be 
concatenated to form a composite measurement vector, which can be used to reconstruct the image of the scene for that particular polarimetric channel. However, this approach yields a very high-dimensional measurement vector (of size $N M \times 1$ ) and a huge dictionary (of size $N M \times Q$ ), which leads to inefficient image reconstruction. Therefore, we apply principal component analysis technique to compress the measurement vector and the dictionary resulting in a compact linear model. Let $\mathbf{C}$ denote the covariance matrix of the new measurements given in (17). Using the eigendecomposition, we can write

$$
\mathbf{C}=\mathbf{G} \boldsymbol{\Lambda} \mathbf{G}^{H}
$$

where $\mathbf{G}=\left[\boldsymbol{g}_{1}, \ldots, \boldsymbol{g}_{R}\right]$ is a matrix of eigenvectors and $\Lambda$ is a diagonal matrix of eigenvalues, arranged in descending order. We define a projection matrix $\mathbf{P}=\left[\boldsymbol{g}_{1}, \ldots, \boldsymbol{g}_{K}\right]$ consisting of the first $(K \ll M)$ eigenvectors; $K$ is determined by using an information theoretic criterion, such as Akaike Information Criterion (AIC) or Minimum Description Length (MDL) [28]. Applying the projection matrix $\mathbf{P}^{H}$ to (17) yields the compressed measurement vector $\widehat{\boldsymbol{x}}_{n, l} \in \mathbb{C}^{K}$,

$$
\widehat{\boldsymbol{x}}_{n, l}=\mathbf{P}^{H} \mathbf{W}^{T} \boldsymbol{\Psi}_{n} \widetilde{\boldsymbol{s}}_{l}=\mathbf{D}_{n} \widetilde{\boldsymbol{s}}_{l} .
$$

Now, we concatenate all compressed measurement vectors belonging to the $l$-th polarimetric channel into a composite vector $\widehat{\mathbf{x}}_{l}=\left[\widehat{\boldsymbol{x}}_{1, l}^{T}, \cdots, \widehat{\boldsymbol{x}}_{N, l}^{T}\right]^{T} \in \mathbb{C}^{N K \times 1}$, and arrange the individual compressed dictionaries into a composite dictionary $\mathbf{D}=\left[\mathbf{D}_{1}^{T}, \cdots, \mathbf{D}_{N}^{T}\right]^{T} \in \mathbb{C}^{N K \times Q}$, to obtain the following compact linear model

$$
\widehat{\mathbf{x}}_{l}=\mathbf{D} \widetilde{s}_{l}
$$

In practice, the formed image consists of outlier pixels that do not form part of the target. To remove these pixels, a smoothing filter $f$ can be applied to the formed image,

$$
\widehat{\boldsymbol{s}}_{l}=\boldsymbol{f} * \widetilde{\boldsymbol{s}}_{l}
$$

where $*$ denotes the convolution operator. The convolution operation can be written in the form of a matrix multiplication, where the filter coefficients are converted into a Toeplitz matrix $\mathbf{F}$, i.e., $\widehat{\boldsymbol{s}}_{l}=\mathbf{F} \widetilde{\boldsymbol{s}}_{l}$. Thus, the smoothing filtering operation can be incorporated into the image formation model as

$$
\widehat{\mathbf{x}}_{l}=\widehat{\mathbf{D}} \widehat{\boldsymbol{s}}_{l},
$$

where $\widehat{\mathbf{D}}=\mathbf{D} \mathbf{F}^{\dagger}$ and $\mathbf{F}^{\dagger}=\left(\mathbf{F}^{T} \mathbf{F}\right)^{-1} \mathbf{F}^{T}$. Using the linear model given in (22) and the mt-BCS technique, we can exploit the inter-channel correlations and the similarity in sparsity support to reconstruct simultaneously the scenes for all polarimetric channels. In the following, we test the proposed CS-based image formation method on real data collected from a stepped-frequency TWRI system.

\section{EXPERIMENTAL RESUlTS}

Real radar measurements are collected in the Radar Imaging Lab of the Center for Advanced Communications at Villanova University. The TWRI scene consists of nine targets: three dihedrals, four trihedrals, a sphere, and a tophat. Each target is placed at a certain distance from the wall. The wall between the radar and the targets is a drywall of thickness $0.127 \mathrm{~m}$, built from a wooden frame with one side fastened with $0.019 \mathrm{~m}$ plywood and the other side with $0.0158 \mathrm{~m}$ gypsum wall board. A stepped-frequency radar of $1 \mathrm{GHz}$ bandwidth centered at $2.5 \mathrm{GHz}$ with a frequency step size of $5 \mathrm{MHz}$ is used to image the targets behind the wall. The transceiver is placed at 69 different positions to synthesize an array aperture of length $1.2246 \mathrm{~m}$. The interested reader is referred to [29] for a detailed description of the experimental setup.

During the experiment, a set of 13,869 space-frequency measurements, i.e., (201 frequencies $\times 69$ antennas) are acquired using the TWRI system. In practice, to reduce the data acquisition time, a subset of frequencies from the selected antennas is collected. In the following experiments, we randomly select a subset of antennas and a subset of frequencies from all polarimetric channels.

In the first experiment, we compare the performance of the wavelet and Fourier dictionary when recovering the radar signal individually and jointly using mt-BCS. The wavelet dictionary $\mathbf{W}$ is obtained using the Daubechies 8 wavelet at four decomposition level. The recovered measurement vector at each antenna location is compared to the full measurement vector, available from the recorded experimental data.

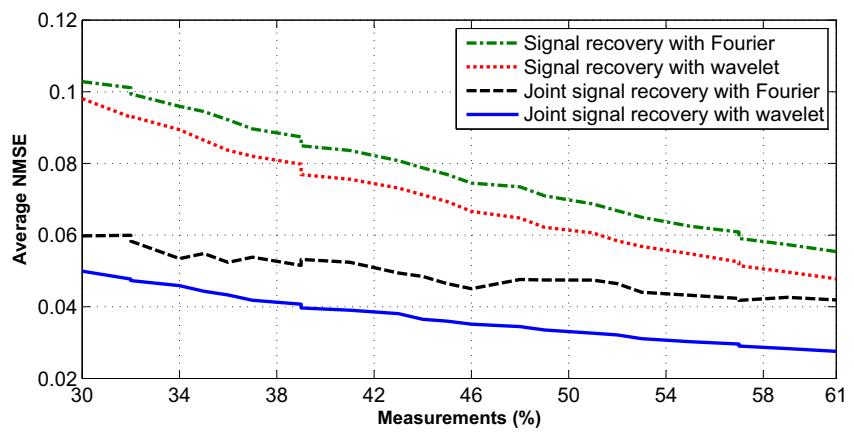

Fig. 1. Average normalized mean square error of the signal recovery as a function of the number of measurements. The result was obtained after 20 trials.

Figure 1 shows the average normalized mean square error (NMSE) as a function of the percentage of the total number of measurements. Using wavelet basis for signal recovery produces lower NMSE compared to Fourier basis. Furthermore, the experimental results illustrated in Fig. 1 show that joint signal recovery gives lower reconstruction error than recovering the radar signal individually. Using $30 \%$ of the full measurement available, the Fourier basis achieves an average NMSE of 0.103 and the wavelet basis produces an NMSE of 0.098 when recovering each signal individually. Jointly recovering the radar signal gives an average NSME of 0.059 for Fourier and 0.05 for wavelet.

In the second experiment, the signals recovered from $35 \%$ of the total measurements are used for scene reconstruction. Here, we compare between individually and simultaneously forming the polarization images. For the smoothing operation, we employ a Gaussian filter of size $3 \times 3$ and a standard 
deviation of 0.6 . For reference, we also apply delay-andsum (DS) beamforming for image formation, where the radar signals are recovered individually. The quality of the formed images is measured using the target-to-clutter ratio (TCR)

$$
\mathrm{TCR}=\frac{1}{N_{\mathrm{t}}} \sum_{q \in A_{\mathrm{t}}}|I(q)|^{2} / \frac{1}{N_{\mathrm{c}}} \sum_{q \in A_{\mathrm{c}}}|I(q)|^{2},
$$

where $|I(q)|^{2}$ is the squared magnitude of the $q$-th complexvalued pixel, $A_{\mathrm{t}}$ is the target region, $A_{\mathrm{c}}$ is the clutter region, $N_{\mathrm{c}}$ and $N_{\mathrm{t}}$ are, respectively, the number of pixels in the clutter and target regions; the clutter region is considered to be the entire image, excluding the target region.

Figure 2 presents the polarization images formed using DS beamforming. Although the subspace projection method mitigates most of the wall reflections, the formed polarization images are still affected by sidelobes and clutter. By contrast, the CS-formed images depicted in Fig. 3, which are formed individually for each channel, contain less background clutter. However, in Fig. 3(c) the targets are barely visible in the cross-polarization image. Figure 4 illustrates the images reconstructed simultaneously using mt-BCS, after joint signal recovery. The average TCR values of the formed images depicted in Figs. 2-4 are listed in Table I. The proposed joint Bayesian CS-based method achieves an average TCR of $103.17 \mathrm{~dB}$, whereas the CS-based method and DS beamforming give average TCRs of $25.15 \mathrm{~dB}$ and $3.6 \mathrm{~dB}$, respectively.

TABLE I

TARGET TO CLUTTER RATIO OF DIFFERENT IMAGE FORMATION METHODS.

\begin{tabular}{|l|c|}
\hline Image formation approach & Average TCR (dB) \\
\hline Proposed joint CS-based method & 103.17 \\
\hline CS-based method & 25.15 \\
\hline DS beamforming & 3.60 \\
\hline
\end{tabular}

\section{CONClusion}

A unified CS framework combining wall clutter mitigation and scene reconstruction was proposed for multi-polarization TWRI. The proposed method employs multitask Bayesian compressed sensing to jointly recover the radar signal from all selected antennas using wavelet dictionary. A wall clutter mitigation technique is applied to the recovered wavelet coefficients. This is followed by dimensionality reduction using PCA to compress the remaining wavelet coefficients so as to produce a compact linear model relating the wavelet coefficients to the image of the scene. Finally, mt-BCS is applied to simultaneously reconstruct all polarization images of the scene. Experimental results showed the proposed method can produce images with reduced background clutter and enhanced targets.

\section{ACKNOWLEDGEMENT}

The authors would like to thank Prof. Moeness Amin and Dr. Fauzia Ahmad from the Center of Advanced Communications at Villanova University, Villanova, PA, USA, for providing the experimental data.

\section{REFERENCES}

[1] M. G. Amin, (Ed.), Through-the-wall radar imaging, Boca Raton, FL: CRC Press, 2010.

[2] F. Ahmad, M. G. Amin, and S. A. Kassam, "Synthetic aperture beamformer for imaging through a dielectric wall," IEEE Transactions on Aerospace and Electronic Systems, vol. 41, no. 1, pp. 271-283, 2005.

[3] F. Ahmad, Y. Zhang, and M. G. Amin, "Three-dimensional wideband beamforming for imaging through a single wall," IEEE Geoscience and Remote Sensing Letters, vol. 5, no. 2, pp. 176-179, 2008.

[4] J. Qian, F. Ahmad, and M. G. Amin, "Joint localization of stationary and moving targets behind walls using sparse scene recovery," Journal of Electronic Imaging, vol. 22, no. 2, pp. 021002(1-10), 2013.

[5] T. Dogaru and C. Le, "Through-the-wall small weapon detection based on polarimetric radar techniques, Tech. Rep. ARL-TR-5014, 2009.

[6] W. Zhang, C. Thajudeen, and A. Hoorfar, "Polarimetric through-thewall imaging," in International Symposium on Electromagnetic Theory (EMTS), 2010, pp. 471-474.

[7] C. Debes, A. Zoubir, and M. Amin, "Enhanced detection using target polarization signatures in through-the-wall radar imaging," IEEE Transactions on Geoscience and Remote Sensing, vol. 50, no. 5, pp. 1968-1979, 2012.

[8] A. Mostafa, C. Debes, and A. Zoubir, "Segmentation by classification for through-the-wall radar imaging using polarization signatures," IEEE Transactions on Geoscience and Remote Sensing, vol. 50, no. 9, pp. 34253439, 2012.

[9] M. Thiel and K. Sarabandi, "Ultrawideband multi-static scattering analysis of human movement within buildings for the purpose of standoff detection and localization," IEEE Transactions on Antennas and Propagation, vol. 59, no. 4, pp. 1261-1268, 2011.

[10] C. H. Seng, A. Bouzerdoum, M. G. Amin, and S. L. Phung, "Two-stage fuzzy fusion with applications to through-the-wall radar imaging," IEEE Geoscience and Remote Sensing Letters, vol. 10, no. 4, pp. 687-691, 2012.

[11] _ , "Probabilistic fuzzy image fusion approach for radar through wall sensing," IEEE Transactions on Image Processing, vol. 22, no. 12, pp. 4938-4951, 2013.

[12] A. Bouzerdoum, J. Yang, and F. H. C. Tivive, "Compressive Sensing for Multi-Polarization Through-Wall Radar Imaging," Compressive Sensing for Urban Radar, Boca Raton, FL: CRC Press, Sept. 2014.

[13] Q. Huang, L. Qu, B. Wu, and G. Fang, "UWB through-wall imaging based on compressive sensing," IEEE Trans. Geoscience and Remote Sensing, vol. 48, no. 3, pp. 1408-1415, 2010.

[14] M. Leigsnering, C. Debes, and A. M. Zoubir, "Compressive sensing in through-the-wall radar imaging," in Proc. IEEE Int. Conf. Acoustics, Speech, and Signal Processing, 2011, pp. 4008-4011.

[15] Y.-S. Yoon and M. G. Amin, "Imaging of behind the wall targets using wideband beamforming with compressive sensing," in Proc. IEEE/SP Workshop on Statistical Signal Processing, 2009, pp. 93-96.

[16] M. G. Amin and F. Ahmad, "Compressive sensing for through-the-wall radar imaging," Journal of Electronic Imaging, vol. 22, no. 3, pp. 1-20, 2013.

[17] V. H. Tang, A. Bouzerdoum, and S. L. Phung, "Two-stage throughthe-wall radar image formation using compressive sensing," Journal of Electronic Imaging, vol. 22, no. 2, pp. (021 006)1-10, 2013.

[18] E. Lagunas, M. G. Amin, F. Ahmad, and M. Najar, "Joint wall mitigation and compressive sensing for indoor image reconstruction," IEEE Transactions on Geoscience and Remote Sensing, vol. 51, no. 2, pp. 891-906, 2013.

[19] Y.-S. Yoon and M. G. Amin, "Spatial filtering for wall-clutter mitigation in through-the-wall radar imaging," IEEE Transactions on Geoscience and Remote Sensing, vol. 47, no. 9, pp. 3192-3208, 2009.

[20] F. H. C. Tivive, M. G. Amin, and A. Bouzerdoum, "Wall clutter mitigation based on eigen-analysis in through-the-wall radar imaging," in Proc. International Conference on Digital Signal Processing, 2011, pp. 1-8.

[21] J. Yang, A. Bouzerdoum, F. H. C. Tivive, and M. G. Amin, "Multiplemeasurement vector model and its application to through-the-wall radar imaging," in Proc. International Conference on Acoustics, Speech and Signal Processing, 2011, pp. 2672-2675.

[22] M. Leigsnering, F. Ahmad, M. G. Amin, and A. M. Zoubir, "Cs based wall ringing and reveberation mitigation for through the wall radar imaging," in IEEE Radar Conference, 2013, pp. 1-5. 


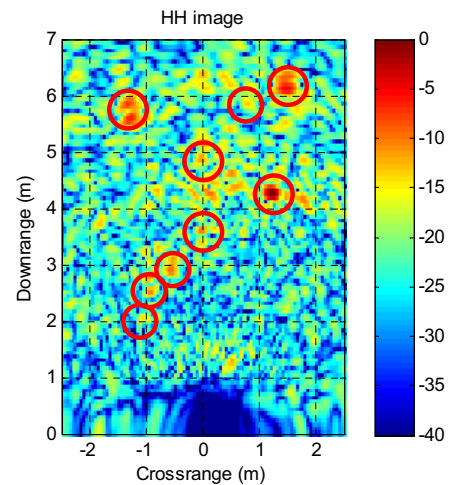

(a)

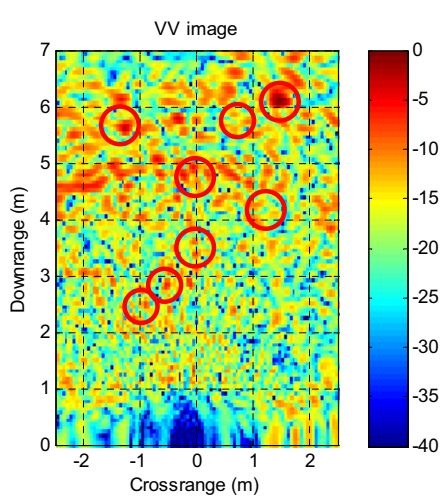

(b)

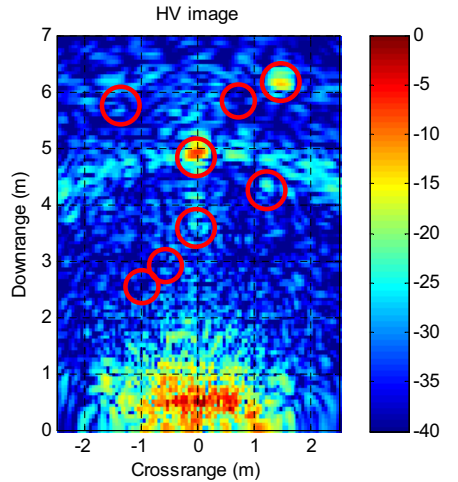

(c)

Fig. 2. Multi-polarization images formed by DS beamforming: (a) HH image, (b) VV image, and (c) HV image.

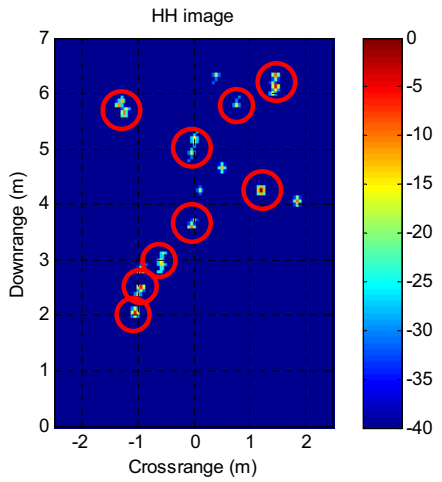

(a)

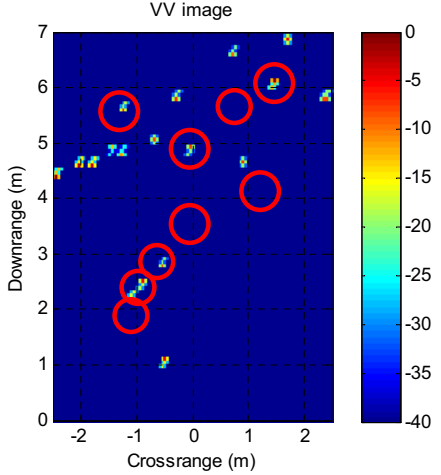

(b)

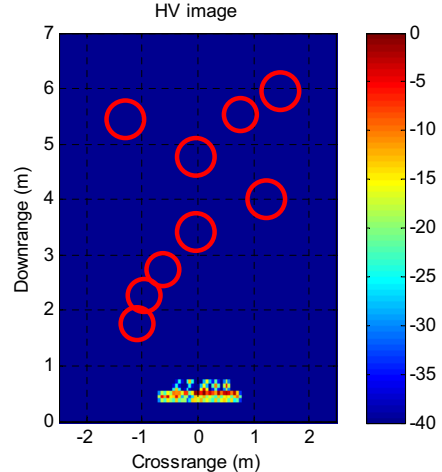

(c)

Fig. 3. Multi-polarization images formed individually by CS-based method: (a) HH image, (b) VV image, and (c) HV image.

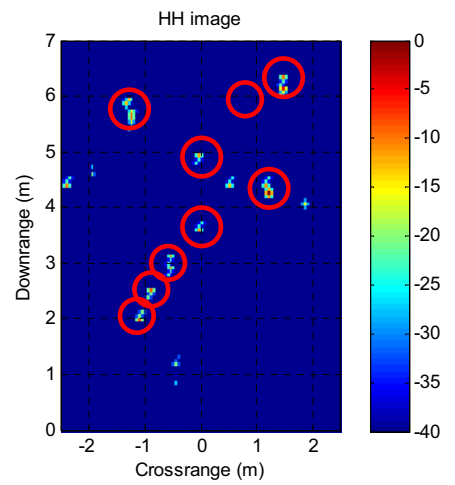

(a)

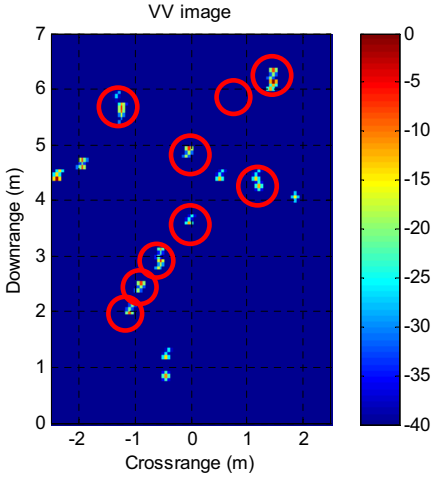

(b)

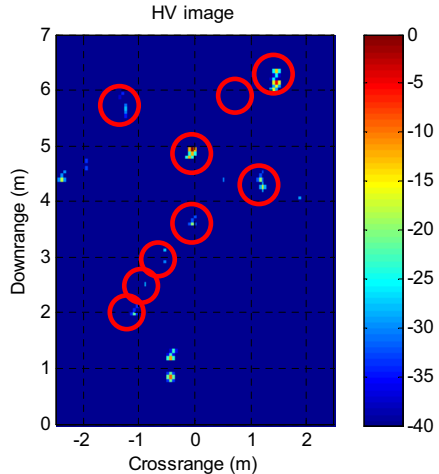

(c)

Fig. 4. Multi-polarization images formed jointly by the proposed Bayesian CS-based method: (a) HH image, (b) VV image, and (c) HV image.

[23] Y.-S. Yoon and M. G. Amin, "Through-the-wall radar imaging using compressive sensing along temporal frequency domain," in IEEE International Conference on Acoustics Speech and Signal Processing (ICASSP), 2010, pp. 2806-2809.

[24] S. Ji, D. Dunson, and L. Carin, "Multitask compressed sensing," IEEE Transactions on Signal Procesing, vol. 57, no. 1, pp. 92-106, 2009.

[25] Q. Wu, Y. D. Zhang, M. G. Amin, and B. Himed, "Complex multitask bayesian compressed sensing," in IEEE International Conference on Acoustics, Speech, and Signal Processing, 2014.

[26] R. Solimene and A. Cuccaro, "Front wall clutter rejection methods in TWI," IEEE Geoscience and Remote Sensing Letters, vol. 11, no. 6, pp. 1158-1162, 2014.
[27] F. H. C. Tivive and A. Bouzerdoum, "An improved SVD-based wall clutter mitigation method for through-the-wall radar imaging," in Proc. IEEE 14th Workshop on Signal Processing Advances in Wireless Communication, 2013, pp. 425-429.

[28] M. Wax and T. Kailath, "Detection of signals by information theoretic criteria," IEEE Transactions on Acoustics Speech Signal Processing, vol. 33, no. 2, pp. 387-392, 1985.

[29] R. Dilsavor, W. Ailes, P. Rush, F. Ahmad, W. Keichel, G. Titi, and M. Amin, "Experiments on wideband through the wall imaging," in Proc. of the SPIE, Vol 5808, Symposium on Defense and Security, Algorithms for Synthetic Aperture Radar Imagery XII Conference, 2005, pp. 196-209. 